\title{
Development of Cartridge-Based Wash-Free Single-Step Plasmonic Enzyme-Linked Immunosorbent Assay Using Poly(vinylpyrrolidinone)-Coated Silver Nanoparticles as a Chromogenic Substrate
}

\author{
Tatsuro Endo, ${ }^{*}$ Kenzo Yamamoto, Kenji Sueyoshi, and Hideaki Hisamoto \\ Department of Applied Chemistry, Graduate School of Engineering, Osaka Prefecture University, \\ 1-1 Gakuen-cho, Naka-ku, Sakai-shi, Osaka, 599-8531, Japan
}

(Received May 30, 2017; accepted August 3, 2017)

Keywords: localized surface plasmon resonance (LSPR), ELISA, nanoparticle, chromogenic substrate

In this study, poly(vinylpyrrolidone) (PVP)-coated silver nanoparticles were used as a chromogenic substrate for the establishment of a colorimetric enzyme-linked immunosorbent assay (ELISA) based on localized surface plasmon resonance (LSPR) called "plasmonic ELISA". The PVP-coated silver nanoparticles exhibit a specific color due to the LSPR. In addition, the LSPR absorbance strength of the PVP-coated silver nanoparticles is changed by the aggregation of the silver nanoparticles which depends on the hydrogen peroxide concentration. From these changes in the LSPR absorbance strength in relation to hydrogen peroxide concentration, plasmonic ELISA was established using a glucose oxidase (GOx)-conjugated secondary antibody and glucose for producing hydrogen peroxide by an enzymatic reaction. Furthermore, on the basis of plasmonic ELISA, a cartridge-based single-step plasmonic ELISA device was fabricated for the realization of a wash-free ELISA system. This cartridge-based ELISA system is constructed with a GOxantibody (GOx-conjugated secondary antibody) release pad, a primary antibody immobilized membrane, a glucose release pad, and a PVP-coated silver nanoparticle-containing hydrogel. By using this construction, the antigen concentration can be determined by introducing only a sample solution. As a result, with this cartridge-based ELISA system, the antigen-antibody reaction was successfully detected at different concentrations of antigen $(0.1-10 \mu \mathrm{g} / \mathrm{ml})$.

\section{Introduction}

Localized surface plasmon resonance (LSPR) is an optical phenomenon that can be observed from noble metal nanostructures such as nanoparticles. ${ }^{(1)}$ Based on LSPR, noble metal nanoparticles exhibit a specific color, which depends on the size, shape, and kind of metal. ${ }^{(2)}$ In addition, using LSPR, biomolecular interactions such as DNA hybridizations and antigen-antibody reactions can be detected with high sensitivity as a change in LSPR optical characteristics such as absorbance strength and peak with the change in the surrounding refractive index. Using these changes in LSPR optical characteristics, highly sensitive label-free biosensors have been widely developed. ${ }^{(3)}$

"Corresponding author: e-mail: endo@chem.osakafu-u.ac.jp http://dx.doi.org/10.18494/SAM.2017.1642 
On the other hand, we revealed that poly(vinylpyrrolidone) (PVP)-coated silver nanoparticles react with hydrogen peroxide, and their LSPR optical characteristics are markedly changed. This change in LSPR optical characteristics is attributed to the chemical reaction between silver and hydrogen peroxide. PVP-coated silver nanoparticles that have reacted with hydrogen peroxide aggregate owing to the chemical reactions. Hence, the LSPR optical characteristics changed depending on the hydrogen peroxide concentration. On the basis of this phenomenon, PVPcoated silver nanoparticles can be applied as a chromogenic substrate. In addition, we have been developing novel sensing methods and devices such as an enzyme sensor using PVP-coated silver nanoparticles. $^{(4-6)}$ From this background, we aimed to apply the PVP-coated silver nanoparticles to an enzyme-linked immunosorbent assay (ELISA). With PVP-coated silver nanoparticles as a chromogenic substrate for the determination of antigen concentration via changes in LSPR optical characteristics, a novel ELISA system called "plasmonic ELISA" is proposed. In addition, in our previous study, the stability of PVP-coated silver nanoparticles was better than these of generically used chromogenic substrates such as 3,3',5,5'-tetramethylbenzidine (TMB) and $o$-phenylenediamine (OPD). Hence, the PVP-coated silver nanoparticle-based ELISA enables us to provide an ELISA system with good stability.

On the other hand, ELISA is widely used for the highly sensitive determination of antigen concentration in the medical field. However, it requires sophisticated liquid handling procedures such as washing and sample introduction. These procedures result in long assay times to determine the antigen concentration with high sensitivity. Hence, to avoid the sophisticated liquid handling procedures and minimize the assay time, we developed a cartridge-based plasmonic ELISA system. This cartridge-based plasmonic ELISA system enables us to determine the antigen concentration by introducing only a sample solution. Previously, we succeeded in developing the element technology for establishing the cartridge-based plasmonic ELISA system. ${ }^{(7)}$ In this study, using the cartridge-based plasmonic ELISA system, the determination of immunoglobulin $\mathrm{G}(\operatorname{IgG})$ by introducing only a sample solution (a single step) was carried out as a model case.

\section{Materials and Methods}

\subsection{Materials}

In this study, for the development of the plasmonic ELISA system, PVP-coated silver nanoparticles (diameter: $20 \mathrm{~nm}$ ) were obtained from Tanaka Kikinzoku Kogyo K.K. (Tokyo, Japan). Acrylamide (AAm), N,N'-methylenebis(acrylamide) (bis-AAm), and 2-hydroxy2-methylpropiophenone (HOMPP) were purchased from Wako Pure Chemical Industries, Ltd. (Osaka, Japan). These were used to prevent the aggregation of PVP-coated silver nanoparticles by the photopolymerization of AAm-hydrogel. Other reagents for the construction of the cartridge-based plasmonic ELISA system were described in our previous study. ${ }^{(7)}$ In addition, ultrapure water $(18.2 \mathrm{M} \Omega \mathrm{cm}$ ) from Sartorius Stedim Biotech (Aubagne, Cedex, France) was used in all the sample preparations. 


\subsection{Apparatus}

Changes in the LSPR optical characteristics were monitored using a 96-well plate reader (Tecan Safire). In addition, for hydrogel photopolymerization, a transilluminator [FAS-III mini (Toyobo Co., Ltd, (Tokyo, Japan)] was used.

\subsection{Construction and detection principle of cartridge-based plasmonic ELISA system}

The construction and a photograph of the cartridge-based plasmonic ELISA system are shown in Figs. 1 and 2, respectively. The fabrication procedure was reported in our previous study. ${ }^{(7)}$ This plasmonic ELISA system was constructed with a glucose oxidase (GOx)-antibody [GOx-conjugated secondary antibody $(30 \mathrm{mg} / \mathrm{ml})$ ] release pad, a primary antibody immobilized membrane, a glucose release pad (9 $\mathrm{mg}$ of glucose), and a PVP-coated silver nanoparticlecontaining hydrogel [AAm and bis-AAm: 8\% (w/v), PVP-coated silver nanoparticles: $10 \mathrm{mg} / \mathrm{ml}$, HOMPP: $1 \%(\mathrm{v} / \mathrm{v})$ ]. In the cartridge, these parts were placed in a glass tube and a Teflon tube (outer diameter: $7 \mathrm{~mm}$, inner diameter: $6 \mathrm{~mm}$ ). By using this construction, the antigen-antibody reaction can be performed by introducing only a sample solution without a washing procedure.

After a sample solution is introduced into the cartridge, the antigen reacts with the GOxantibody. Then, the GOx-antibody-reacted antigen is captured in the primary antibody immobilized membrane. On the other hand, the antigen-unreacted free GOx-antibody passes through the antibody-immobilized membrane. Subsequently, the GOx of the free GOx-antibody reacts in the glucose release pad, which depends on the free GOx-antibody concentration (related with antigen concentration). Finally, the hydrogen peroxide produced by the enzymatic reaction between glucose and GOx reacts with PVP-coated silver nanoparticles in the PVP-coated silvernanoparticles containing hydrogel. As a result, the LSPR optical characteristics that depend on antigen concentration can be determined.

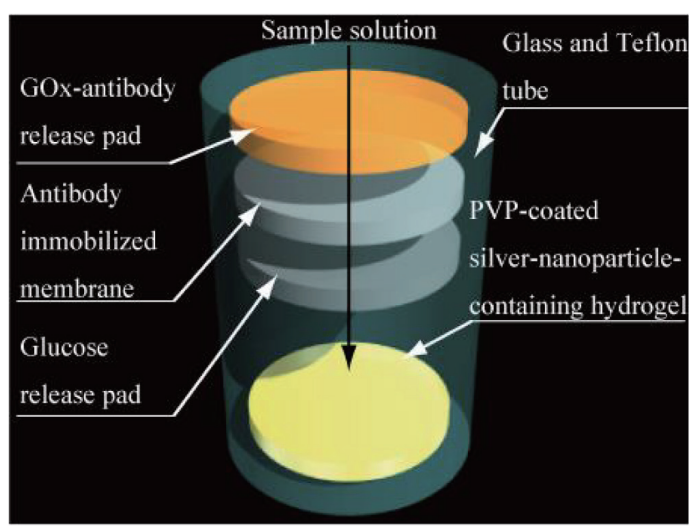

Fig. 1. (Color online) Schematic illustration of construction of cartridge-based plasmonic ELISA system using PVP-coated silver nanoparticles.

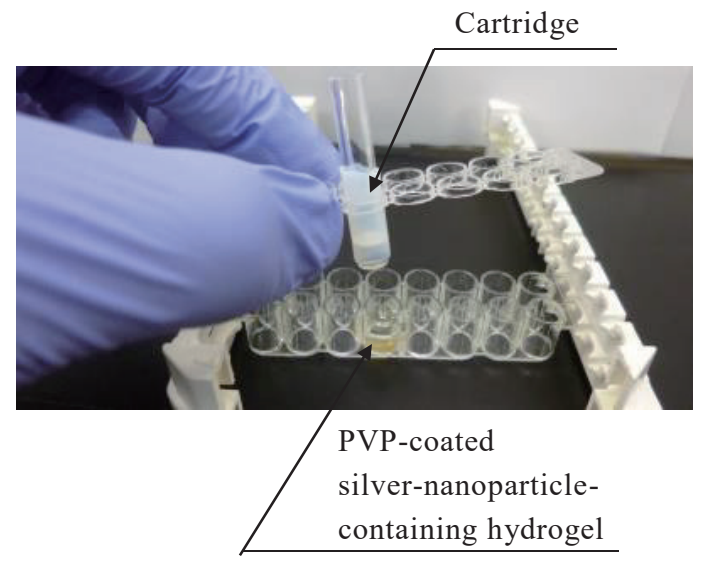

Fig. 2. (Color online) Photograph of cartridgebased plasmonic ELISA system. 
In our previous study, we optimized the experimental conditions. ${ }^{(7)}$ From these optimized conditions, in this study, the antigen concentration dependence on the change in LSPR optical characteristics was evaluated.

\subsection{Determination of antigen concentration using cartridge-based plasmonic ELISA system}

For the evaluation of antigen concentration dependence using a cartridge-based plasmonic ELISA system, anti-human IgG antibody was immobilized onto the primary antibody immobilized membrane. Then, the cartridge-based plasmonic ELISA system was placed in the 96-well plate. Simultaneously, different concentrations of human IgG solutions $(0-10 \mu \mathrm{g} / \mathrm{ml}, 300 \mu \mathrm{l})$ were introduced into the cartridge-based plasmonic ELISA system for $30 \mathrm{~min}$ at room temperature. Finally, the change in the LSPR optical characteristics were monitored using a 96-well plate reader at $408 \mathrm{~nm}$, the LSPR absorbance peak of the PVP-coated silver nanoparticles (Fig. 3).

\section{Results and Discussion}

\subsection{Time-dependent change in LSPR optical characteristics using cartridge-based plasmonic ELISA system}

The time-dependent change in the LSPR optical characteristics at $408 \mathrm{~nm}$ following the introduction of different concentrations of antigen solutions is shown in Fig. 4. As a result, the concentration dependence was determined using the cartridge-based plasmonic ELISA system. In addition, by introducing an antigen solution, the LSPR optical characteristics were changed immediately. By introducing the antigen solutions, GOx-antibody-reacted antigen was immediately captured by the immobilized antibody on the membrane. Furthermore, the free GOxantibody passed through the primary antibody immobilized membrane. Hence, the LSPR optical characteristics were rapidly changed depending on the antigen concentration by hydrogen peroxide owing to the enzymatic reaction between GOx and glucose.

From the time dependent change in the LSPR optical characteristics, differences in the change in the LSPR absorbance strength were found to be small. From our previous study, we determined

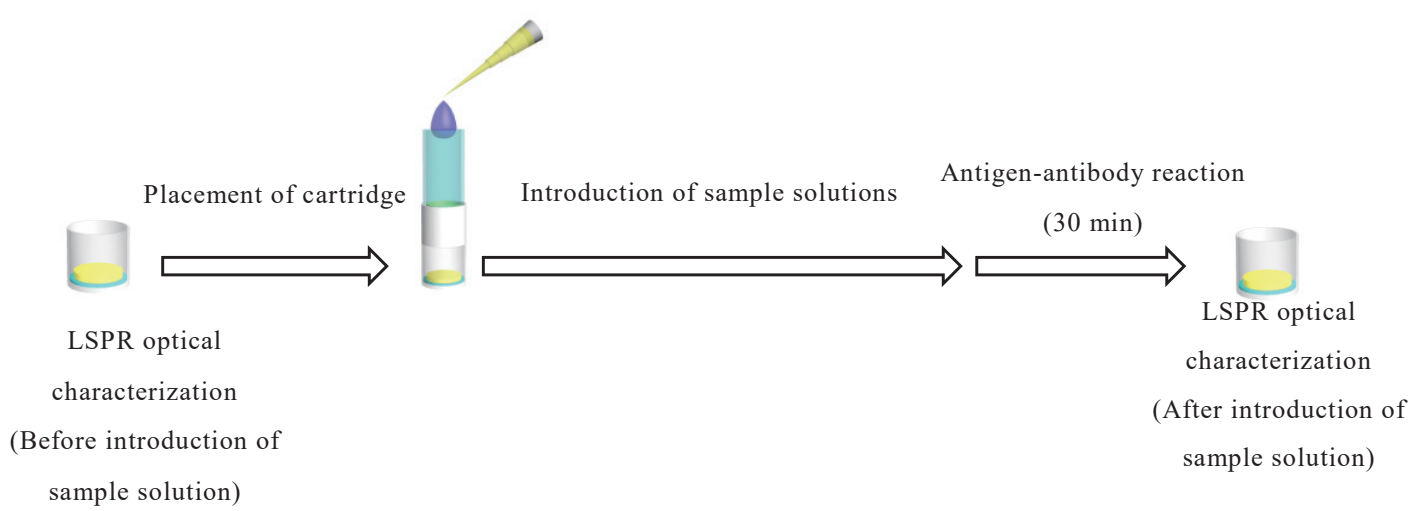

Fig. 3. (Color online) Experimental procedure using cartridge-based plasmonic ELISA system. 
that the steric hindrance of the antibody because of the conjugation of GOx affects the antigenantibody reaction efficiency. ${ }^{(7)}$ When the GOx was conjugated near the antigen-binding fragment (Fab) region, the reaction efficiency was markedly decreased between the antigen and the antibody. As a result, the free GOx-antibody concentration increased. Hence, we expected that the differences in the changes in the LSPR absorbance strength were decreased.

In addition, from the time dependent of change in LSPR optical characteristics, a decay constant of $0.138 \mathrm{~s}^{-1}$ was calculated. Compared with the previous reports based on enzymatic reactions, this value is extremely long. The rate of diffusion in the hydrogel affects this long decaying constant.

\subsection{Evaluation of antigen concentration dependence using cartridge-based plasmonic ELISA system}

The antigen concentration dependence for cartridge-based plasmonic ELISA system is shown in Fig. 5. From these results, using the cartridge-based plasmonic ELISA system, we could successfully detect $0.1 \mu \mathrm{g} / \mathrm{ml}$ of antigen. Using this system, antigens can be detected easily without a washing procedure such as those required in immunochromatographic assays. ${ }^{(8)}$ However, the detection limit of this system is insufficient for detecting the biomarkers of cancers ${ }^{(9)}$ and diabetes. ${ }^{(10)}$ To realize the highly sensitive detection, improvement of the following points is required.

(1) Hydrogel

In this study, to prevent the aggregation of PVP-coated silver nanoparticles, a hydrogel was prepared. However, the diffusion time of hydrogen peroxide into the hydrogel is long. Hence, to reduce the diffusion time, a different hydrogel component or another material is needed.

(2) Antibody immobilized membrane

To increase the level of difference in the change in LSPR absorbance strength with antigen concentration, the retention time of antigen and GOx-antibody in the primary antibody immobilized membrane should be increased. To increase the retention time in the primary antibody immobilized membrane, the membrane pore size of the material needs to be investigated.

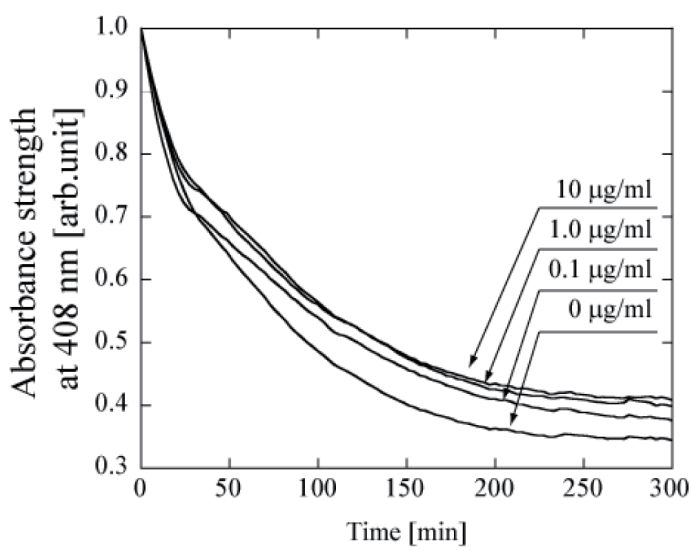

Fig. 4. Time-dependent change in LSPR optical characteristics following the introduction of different concentrations of antigen solutions.

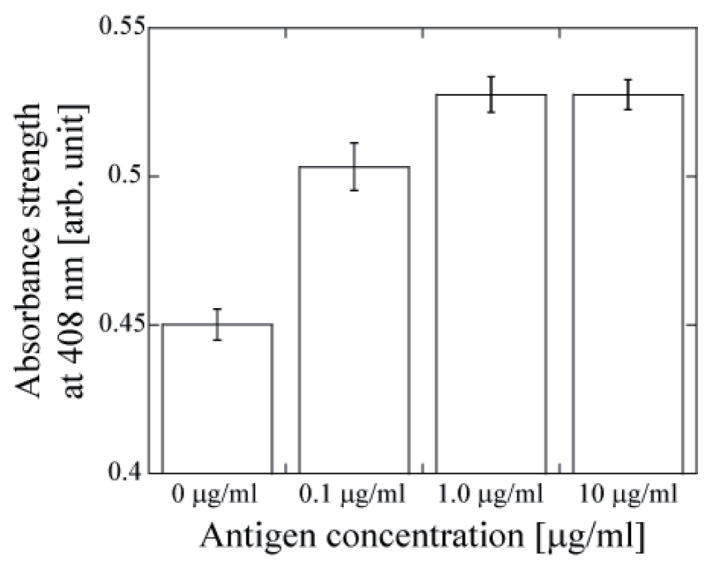

Fig. 5. Dependence of absorbance on antigen concentrations using cartridge-based plasmonic ELISA system. 
(3) Hydrogel design

In this study, a large PVP-coated silver-nanoparticle-containing hydrogel was prepared (diameter: $6 \mathrm{~mm}$ ). By preparing a smaller hydrogel, the reaction efficiency will be improved because of the increase in the surface area.

\section{Conclusions}

In this study, PVP-coated silver nanoparticles were used as a chromogenic substrate for the development of a novel ELISA system called "plasmonic ELISA". In addition, a cartridge-based plasmonic ELISA system was established, and the antigen-antibody reaction was successfully detected by introducing only a sample solution. However, for the highly sensitive detection of antigen, further investigations are required. Using this plasmonic ELISA system, the antigen concentration can be determined using a cost-effective optical setup such as a smartphone and a digital camera in the future.

\section{Acknowledgments}

This work was supported by a Grant-in-Aid for Scientific Research (B) (15H03009).

\section{References}

1 L. J. Sherry, R. Jin, C. A. Mirkin, G. C. Schatz, and R. P. Van Duyne: Nano Lett. 6 (2006) 2060.

2 T. R. Jensen, M. D. Malinsky, C. L. Haynes, and R. P Van Duyne: J. Phys. Chem. B 104 (2000) 10549.

3 E. Petryayeva and U. J. Krull: Anal. Chim. Acta 706 (2011) 8.

4 T. Endo, A. Shibata, Y. Yanagida, Y. Higo, and T. Hatsuzawa: Mater. Lett. 64 (2010) 2105.

5 T. Endo, Y. Yanagida, and T. Hatsuzawa: Measurement 41 (2008) 1045.

6 T. Endo, R. Ikeda, Y. Yanagida, and T. Hatsuzwa: Anal. Chim. Acta 61 (2008) 205.

7 K. Yamamoto, K. Sueyoshi, H. Hisamoto, and T. Endo: Electron. Commun. Jpn. 100 (2017) 1307.

8 M. G. Weller: Fresenius J. Anal. Chem. 366 (2000) 635.

9 C. M. Ferrier, H. H. de White, H. Straatman, D. H. Van Tienoven, W. L. Van Geloof, F. J. R. Rietveld, D. G. J. Sweep, D. J. Ruiter, and G. N. P. Van Muijen: Br. J. Cancer 79 (1999) 1534.

10 T. C. Vukovich and G. Schernthaner: Diabetes 35 (1986) 617.

11 T. Tao, R. A. Raffel, A.-K. Souid, and J. Goodisman: Biophys. J. 86 (2009) 2977.

12 M.Wu, Z. Lin, and O. S. Wolfbeis: Anal. Biochem. 320 (2003) 129. 